\title{
Correspondence
}

(The Editors do not hold themselves responsible for opinions expressed by correspondents)

\section{Cerebral sparganosis}

The Editor - Sir,

I read the recent publication on cerebral sparganosis by Chiu et al [1] with great interest. The authors reported on the magnetic resonance (MR) spectroscopy and perfusion character of this neurological disorder. This neurological parasitic infestation is extremely rare [2] and there is still no clear conclusion on the clinical usefulness of neuroimaging for its diagnosis. Indeed, in a large case series of sparganosis from Thailand, the final diagnosis of cerebral sparganosis was not obtained from neuroimaging [2]. The mass lesion demonstrated by MR imaging did not bring any conclusive diagnosis and removal of granuloma from the identified pathological site was necessary to obtain the final definitive diagnosis by parasitological examination $[2,3]$.

In the endemic area of sparganosis, where other neurological parasitic infestations (e.g. cysticercosis and gnathostomiasis) are also common, the clinical usefulness of MR imaging is very limited in providing a definitive diagnosis of cerebral sparganosis. A history of risky behaviour (e.g. drinking impure water, eating frog or snake meat, or using frog or snake meat as a poultice) might be a clue and offers supporting evidence for a presumptive diagnosis in cases of abnormal brain MR imaging results [2].

Yours etc.,

V WIWANITKIT

Wiwanitkit House

Bangkhae

Bangkok

Thailand 10160

E-mail: wviroj@yahoo.com

(Received 21 April 2010, Accepted 26 May 2010)

DOI: $10.1259 / \mathrm{bjr} / 22825470$

\section{References}

1. Chiu CH, Chiou TL, Hsu YH, Yen PS. MR spectroscopy and MR perfusion character of cerebral sparganosis: a case report. Br J Radiol 2010;83:e31-4.

2. Wiwanitkit V. A review of human sparganosis in Thailand. Int J Infect Dis 2005;9:312-6.

3. Bo G, Xuejian W. Neuroimaging and pathological findings in a child with cerebral sparganosis. Case report. J Neurosurg 2006;105:470-2. 